\title{
Relationship between the shape of the obturator foramen and the shape of the pelvic cavity in adult women
}

\author{
Ivan Vasilyevich Gaivoronskiy ${ }^{1,2}$ (D), Ivan Antonovich Labetov ${ }^{3}$ (D), Gleb Valerevich Kovalev ${ }^{1,3}$ (D),

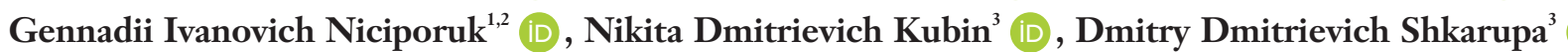 \\ ${ }^{1}$ Department of General Anatomy, "Military Medical Academy named after S.M. Kirov" of the Ministry of Defense of the Russian Federation, \\ St. Petersburg, Russian Federation \\ ${ }^{2}$ Department of Morphology, St. Petersburg State University, St. Petersburg, Russian Federation \\ ${ }^{3}$ Department of Urology, Clinic of High Medical Technology named after N.I. Pirogov, St. Petersburg State University, St. Petersburg, Russian Federation
}

\begin{abstract}
Objectives: There is variable information on the efficiency of surgical treatment of urinary incontinence (SUI). To date, there is no common understanding of the role of variations in the pelvic anatomy for the success of surgical treatment of SUI. The aim of the present study was to investigate the relationship between the morphometric characteristics of the pelvis, and particularly the shape of the obturator foramen with the shape of pelvic cavity, in adult women.

Methods: Sixty-one articulated specimens of female pelvis from the bone collection of the Museum of the St. Petersburg Military Medical Academy named after S.M. Kirov were studied. A pelviometric form was developed, according to the linear and angular parameters of the pelvis and the obturator foramen, and indices of the pelvic cavity and the obturator foramen were evaluated. The discriminant analysis was used to determine the parameters that have the greatest effect on the shape of the obturator foramen.

Results: Based on the calculated indices of the pelvic cavity, the form of the pelvic cavity was determined as: narrowing downwards, cylindrical, and expanding downwards. For pelviometric parameters, for each form of the pelvic cavity, significant differences were defined, and a wide range of anatomical variations anatomy were also demonstrated. A mathematical model for predicting the shape of the obturator foramen was developed with the help of the discriminant analysis. At the same time, two main forms of the obturator foramen were marked out as triangular and ellipsoid. The triangular form was predominantly found in cylindrical and narrowing downwards pelvis groups, and the ellipsoid form dominated in the expanding downwards group.

Conclusion: Longitudinal and transverse indices of the pelvic cavity may allow us to determine its shape as narrowing downwards, cylindrical or expanding downwards. The obturator foramen has a typical shape in each particular configuration of the pelvis.
\end{abstract}

Keywords: discriminant analysis; lesser pelvic cavity; morphometry; obturator foramen; pelvic anatomy; pelvimetry; stress urinary incontinence

Anatomy 2020;14(1):53-60 @2020 Turkish Society of Anatomy and Clinical Anatomy (TSACA)

\section{Introduction}

The human pelvis has been of interest for many years. There are a large number of studies on the development, functional role and morphological variations of pelvic bones. ${ }^{[1,3]}$ However, even today the detection of new anatomical relationship does not lose its relevance for surgical correction of the pelvic floor dysfunction. One of the most popular and studied methods of treatment of stress urinary incontinence in women is the implantation of synthetic suburethral sling installed in structures located in the area of the obturator foramen. However, information about its efficiency varies greatly, from 50 to $100 \%$. According to a meta-analysis study conducted in 2017 by Maggiore et al., ${ }^{[4]}$ the objective effectiveness of 
transobturator sling is $67 \%$. Today, there is a problem with the installation of the sling, which consists in the absence of a personalized approach based on the variations in pelvis anatomy. ${ }^{[4,5]}$ These data together with the results of the studies of anatomical variability of the pelvis imply the possibility of improving the surgical technique, taking into account the individual anatomical features of the obturator foramen and neighboring area. ${ }^{[6,7]}$ The impact of pelviometric parameters as well as the variability of the shape of the pelvis on the development of pelvic floor dysfunction are of interest for obstetric and gynecological practice. ${ }^{[8-10]}$ Nevertheless, there are no studies evaluating the relationship of morphometric characteristics of some pelvic structures involved in surgical interventions with the shape of pelvic cavity. Thus, the aim of the present study was to investigate the relationship between the morphometric characteristics of the pelvis, particularly the shape of the obturator foramen and the shape of pelvic cavity in adult women.

\section{Materials and Methods}

Sixty-one articulated pelvises selected from the Gruber collection were studied. The collection which consists of 1200 human skeletons is located in the museum of the Department of General Anatomy, Military Medical Academy named after S.M. Kirov. For each skeleton, the ethnic group, age, height, weight and other anthropometric characteristics were documented. The pelvises of Caucasian women aging from 35 to 65 were randomly selected for the study. Damaged samples were excluded from the study.

A pelviometric form was developed for the study, on the basis of which the linear and angular parameters of the pelvis were evaluated. Additionally, the lumbosacral angle was measured. A total of 21 parameters were studied. These parameters were:

- Direct size of pelvic inlet: the distance in the median plane between the upper inner edge of the pubic symphysis and the mid-point of sacral promontory (A);

- Direct size of pelvic outlet: the distance between the lower internal edge of the pubic symphysis and the apex of the coccyx (B);

- Longitudinal size of the pelvic cavity: the distance between the lower edge of the pubic symphysis and the apex of the sacrum (Spc);

- Transverse size of the pelvic inlet: the distance between the furthest points of the arched lines in the frontal plane $(\mathrm{C})$;
- Transverse size of the pelvic outlet: the distance between the inner surfaces of the ischial tuberosities (D);

- Symphysis-tuberosity distance: the distance between the lower edge of the symphysis and the most distant points of the lower inner edges of the ischial tuberosities (Std);

- Pelvic height: the distance between the highest point of the iliac crest and the lowest point of the ischial tuberosity (right, left) $(\mathrm{Ph})$;

- Height of pubic symphysis: the distance between upper and lower edges of pubic symphysis (Hps);

- Thickness of pubic symphysis: the largest distance between the anterior and posterior surfaces of the symphysis in the median plane (Tps);

- Interspinal distance: the distance between the anterior superior iliac spines (Is);

- Width of pubic symphysis: the distance between symphysis surfaces in the middle third (Wps);

- Interobturator foramina distance: the smallest distance between the obturator foramina (Ifd);

- Subpubic angle: the angle which formed at pubic arch by the convergence of the inferior rami of the ischium and pubis on either side ${ }^{\circ}(\mathrm{Sa})$;

- Length of sacrospinous ligament: the distance from sacrum to spina ischiadica (Lssl);

- Length of sacrotuberous ligament: the distance from sacral apex to ischial tuberosities (Lstl);

- Lower angle of the obturator foramen: the angle formed by the branches of pubic and ischial bones, forming the lower boundary of the obturator foramen ${ }^{\circ}$ (Laof);

- The medial angle of the obturator foramen: the angle formed by the pubic bone branches, forming the medial boundary of the obturator foramen ${ }^{\circ}$ (Maof);

- The width of the obturator foramen: the distance between the furthest points on the side edges of the obturator foramen (Wof);

- The length of the obturator foramen: the distance between the highest point on the top edge of the obturator foramen and the lowest point on its lower edge (Lof);

- The depth of the obturator foramen: the distance from the lateral edge of the acetabulum, perpendicular to the point on the inner surface of the pubic bone (Dof);

- Lumbosacral angle: the angle formed between a line across the plane of the superior margin of the sacrum and a horizontal line ${ }^{\circ}$ (Lsa). 
The pelvic indices (longitudinal-longitudinal and transverse-transverse) were used to determine the shape of the pelvic cavityas narrowing downwards, expanding downwards and cylindrical (Figure 1). Longitudinal-longitudinal (LL) index represents the ratio of the direct size of the pelvic inlet (Figure 2a) to the direct size of the outlet (Figure 2b). The transverse-transverse (TT) index, respectively represents the ratio of the transverse size of the pelvic inlet to the transverse size of the outlet (Figure 2). The obturator foramen index (OFI) was determined as the ratio of the width of the obturator foramen (Wof) to the length of the obturator foramen (Lof). On the basis of visual assessment three forms of obturator foramen were identified: triangular, ellipsoid and quadrangular
(Figure 3) which were subsequently subjected to discriminant analysis. A discriminant analysis was applied to classify the forms of the obturator foramen, which has a number of advantages over similar mathematical methods, namely: takes into account the variability of the parameter and considers a set of available indicators, which reflect the specific weight of the influence of each measured parameter to determine the shape of the obturator foramen.

The measurements of pelviometric parameters have been made with the use of Martin pelvimeter, measuring ruler, protractor. Because there were no significant differences between right and left sides, the mean measure- a

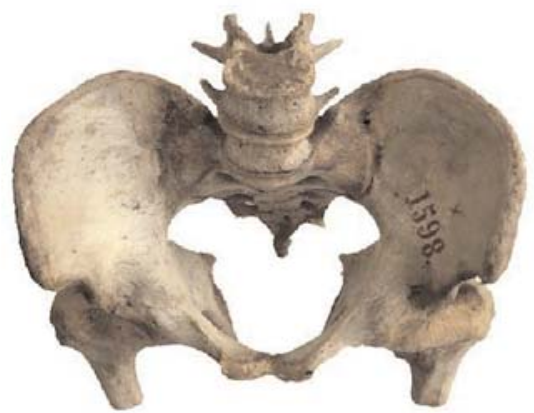

b

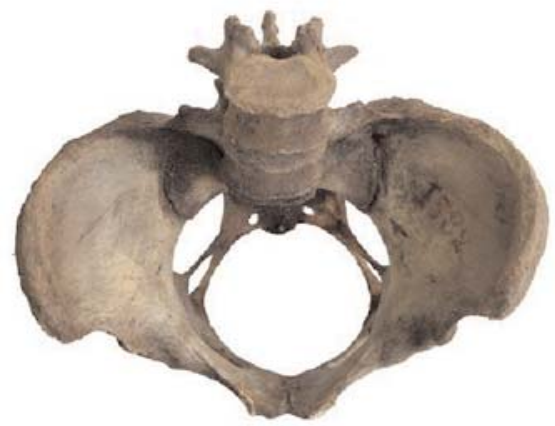

C

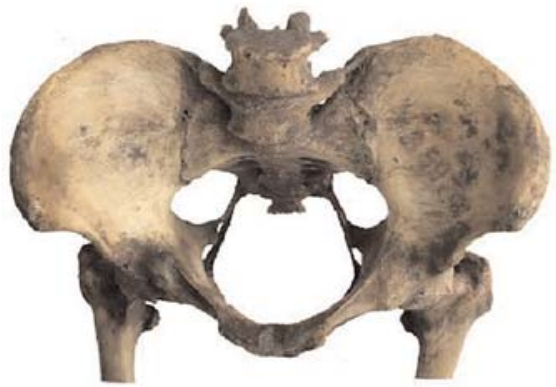

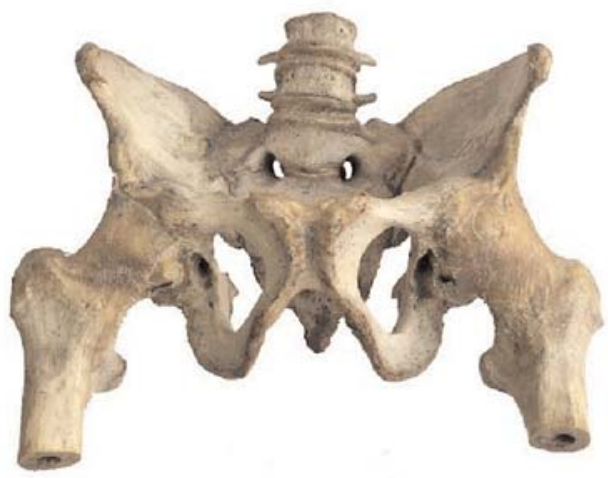
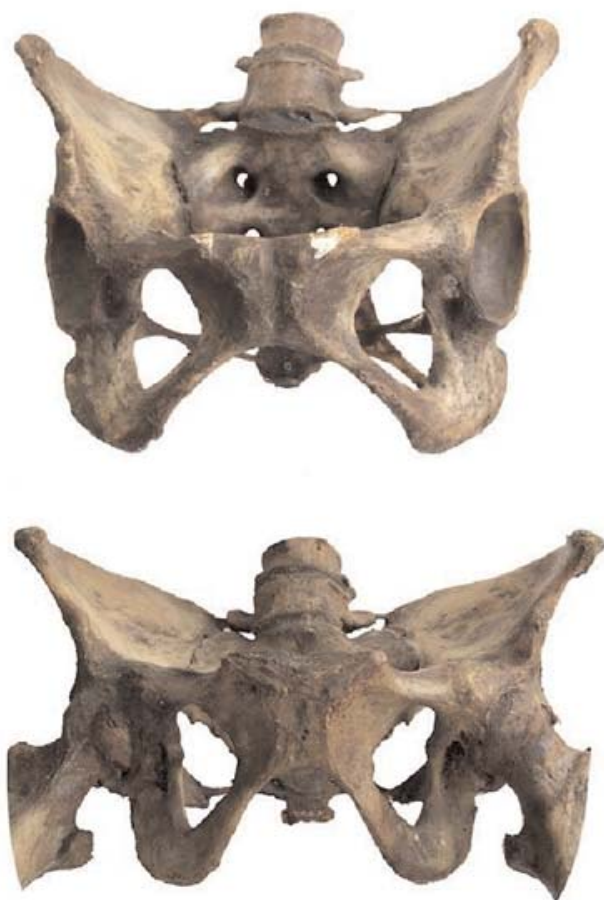
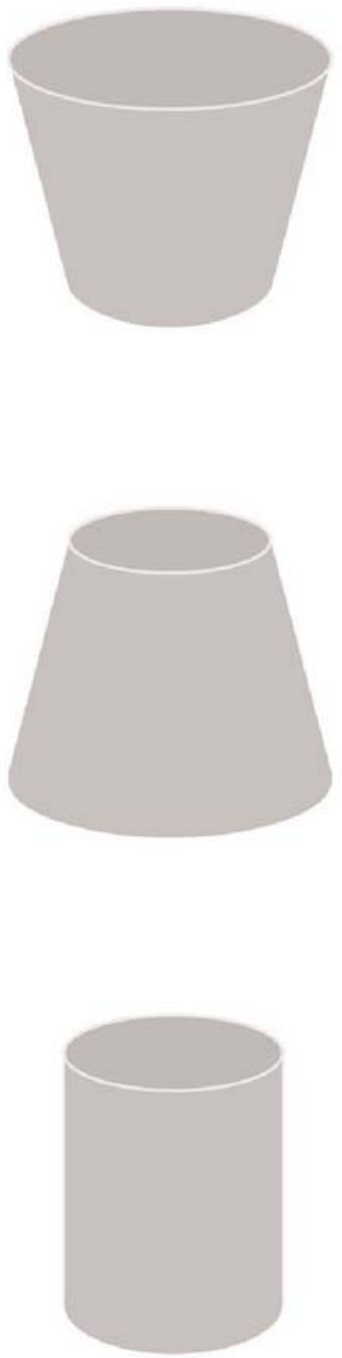

Figure 1. Variation of lesser pelvic cavity (a) narrowing downwards; (b) expanding downwards; (c) cylindrical. 

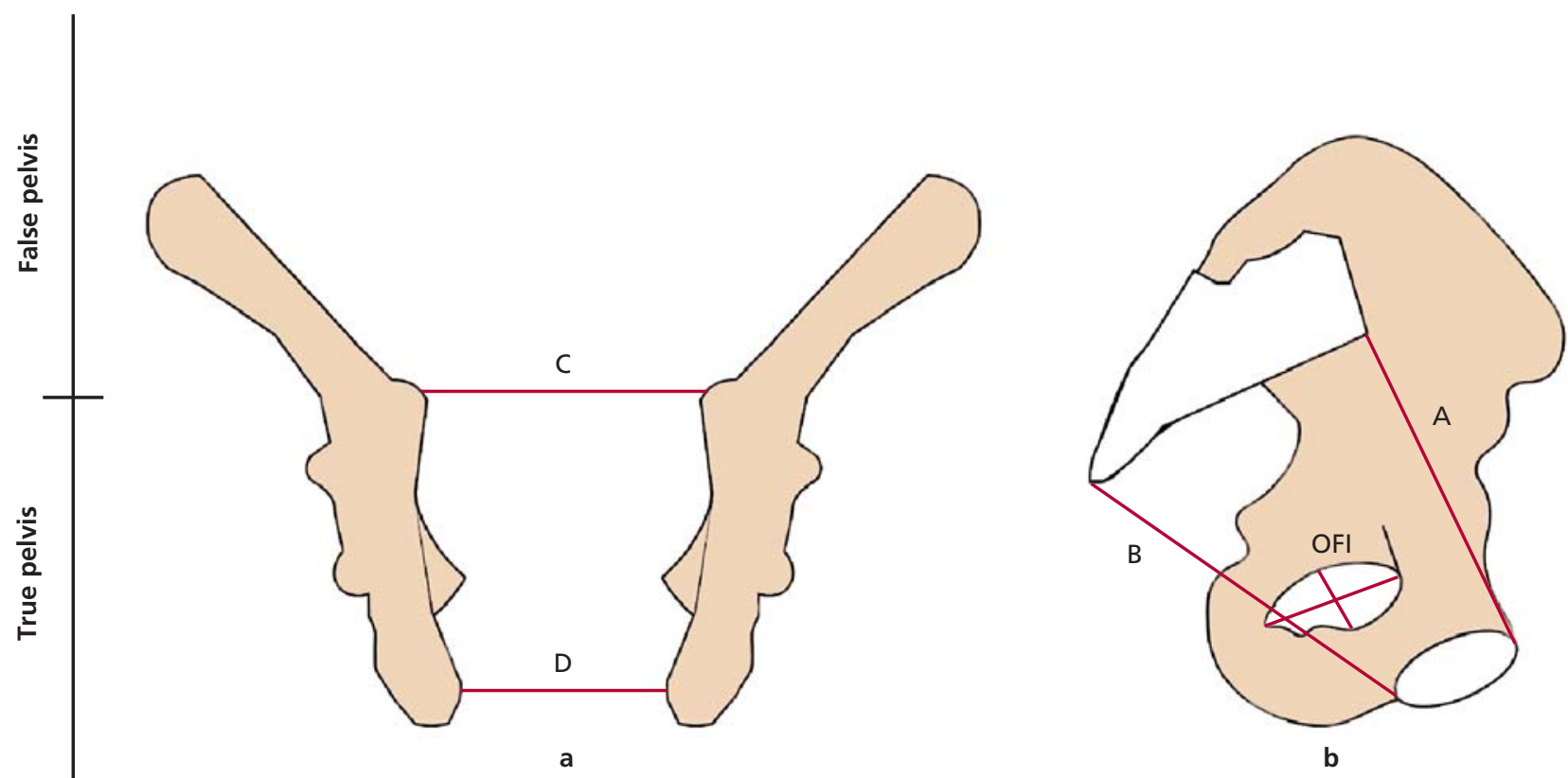

Figure 2. (a) Coronal section of the pelvis and (b) medial view of the left hip bone articulated with the sacrum. A: direct size of the pelvic inlet; B: direct size of the pelvic outlet; C: transverse size of the pelvic inlet; D: transverse size of pelvic outlet; OFI: width of the obturator foramen to the length of the obturator foramen.
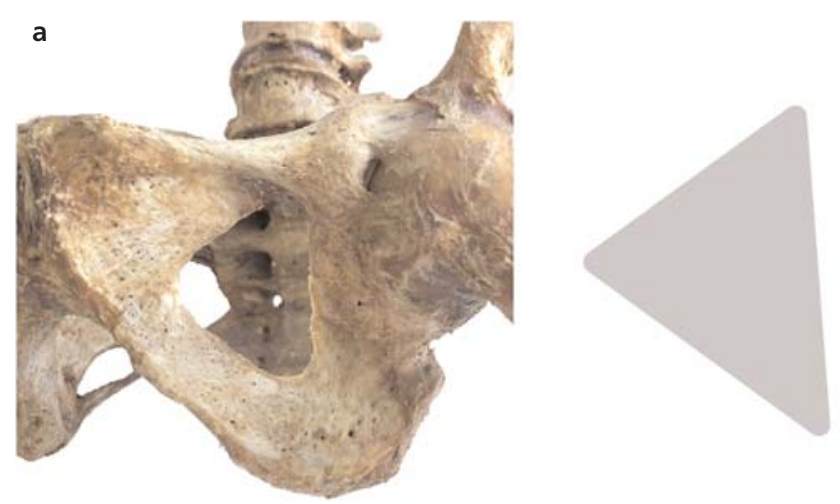

b
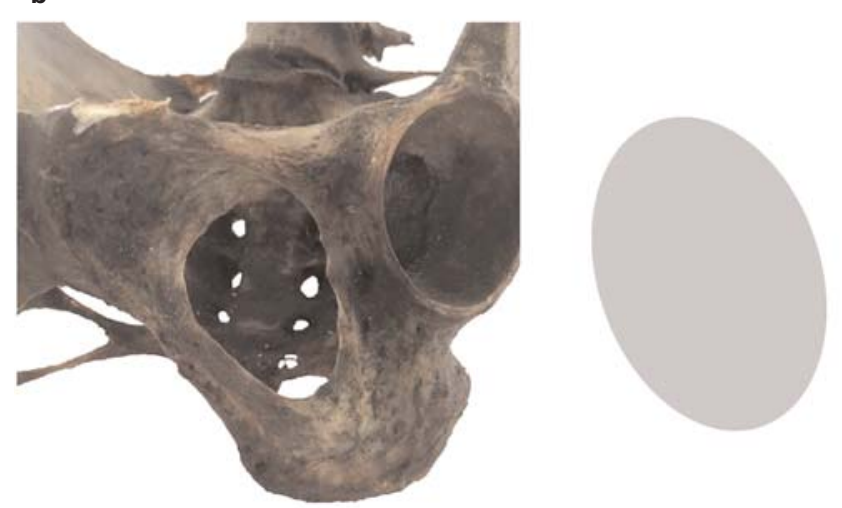

Figure 3. Shapes of obturator foramen of female pelvis. (a) triangular; (b) ellipsoid. ments were considered. Statistical analysis of the obtained data was evaluated using STATISTICA v10 (Dell Statistica, Tulsa, OK, USA) software. The criterion of statistical significance of the tested hypotheses was the value $\mathrm{p}<0.05$. To analyze the qualitative parameters defined with frequencies and percentages, the chi-square criterion and Fisher's exact test were used. Normalities were tested using the Shapiro-Wilk criterion. The values were defined with the mean value and standard error of the mean (in case of normal distribution) or by the median and quantile otherwise. The non-parametric KruskalWallis criterion was used to check the differences between the groups.

This study was approved by the local research ethics committee of Military Medical Academy named after S. M. Kirov meeting dated 22.03.2019 (No:199).

\section{Results}

The values of linear dimensions in centimeters $(\mathrm{cm})$ and angular characteristics in degrees $\left(^{\circ}\right)$ were are presented in Table 1. Parameters A, B, Spc, C, D, Std, Tps, Sa, Laof, Maof, Lsa (Parameter no: 1,2,3,4,5,6,9,13,16,17 and 21 in Table 1) differed significantly with the coefficient of variation more than $20 \%$. Considering the high variability of these parameters, they were selected as the most important parameters for classification of the pelvises. 
Table 1

Pelviometric form: parameters of the true pelvis and the obturator foramen.

\begin{tabular}{|c|c|c|c|c|}
\hline \multirow[b]{2}{*}{ Pelviometric parameters $(\mathrm{cm}) /\left(^{\circ}\right)$} & \multicolumn{3}{|c|}{ Values } & \multirow{2}{*}{$\begin{array}{l}\text { Coefficient of } \\
\text { variation (\%) }\end{array}$} \\
\hline & Min & Max & Mean \pm SD & \\
\hline Direct size of pelvic (A) & 8.5 & 12.8 & $11.1 \pm 1.4$ & 22.76 \\
\hline Direct size of pelvic outlet (B) & 7.1 & 12 & $8.8 \pm 1.1$ & 25.27 \\
\hline Longitudinal size of the pelvic cavity (Spc) & 8.8 & 13.4 & $11.9 \pm 0.8$ & 20.32 \\
\hline Transverse size of the true pelvic inlet (C) & 11.5 & 14.5 & $13.1 \pm 0.8$ & 24.43 \\
\hline Transverse size of the pelvic outlet (D) & 7.5 & 12.5 & $10.3 \pm 1.1$ & 23.29 \\
\hline Symphysis-tuberosity distance (Std) & 8.2 & 10.6 & $9.4 \pm 0.5$ & 22.46 \\
\hline Pelvic height (right, left) (Ph) & $\begin{array}{l}17.4(\mathrm{R}) \\
17.6(\mathrm{~L})\end{array}$ & $\begin{array}{l}22.4 \\
22.5 \\
\end{array}$ & $\begin{array}{l}19.4 \pm 1.1 \\
18.9 \pm 1.9 \\
\end{array}$ & $\begin{array}{l}5.62 \\
6.11 \\
\end{array}$ \\
\hline Height of pubic symphysis (Hps) & 2.9 & 4.4 & $3.5 \pm 0.3$ & 8.96 \\
\hline The pubic symphysis thickness (Tps) & 1.0 & 2.1 & $1.5 \pm 0.3$ & 22.79 \\
\hline Interspinal distance (Is) & 19.0 & 25.8 & $23.6 \pm 1.5$ & 17.71 \\
\hline Width of pubic symphysis (Wps) & 0.7 & 1.3 & $1.0 \pm 0.2$ & 5.84 \\
\hline Interobturator foramina distance (Ifd) & 3.9 & 6.3 & $5.2 \pm 0.6$ & 9.6 \\
\hline Subpubic angle ${ }^{\circ}(\mathrm{Sa})$ & 48 & 117 & $78.3 \pm 9.1$ & 21.76 \\
\hline Length of sacrospinous ligament (Lssl) & 4.5 & 6.1 & $5.2 \pm 0.3$ & 13.94 \\
\hline Length of sacrotuberous ligament (Lstl) & 5.6 & 8.7 & $7.1 \pm 1.2$ & 11.27 \\
\hline Lower angle of the obturator foramen ${ }^{\circ}$ (Laof) & 49.0 & 80.0 & $59.2 \pm 11.1$ & 24.34 \\
\hline The medial angle of the obturator foramen ${ }^{\circ}$ (Maof) & 71.0 & 95.5 & $80.4 \pm 9.8$ & 20.55 \\
\hline The width of the obturator foramen (Wof) & 2.6 & 4.3 & $3.4 \pm 0.5$ & 12.94 \\
\hline The length of the obturator foramen (Lof) & 4.0 & 5.9 & $4.9 \pm 0.6$ & 7.96 \\
\hline The depth of the obturator foramen (Dof) & 1.3 & 2.4 & $1.8 \pm 0.3$ & 9.12 \\
\hline Lumbosacral angle ${ }^{\circ}$ (Lsa) & 119.7 & 143.2 & $136.4 \pm 7.1$ & 21.19 \\
\hline
\end{tabular}

The TT index was $1.32 \mathrm{~cm}$ on average (min: $0.95 \mathrm{~cm}$, max: $1.5 \mathrm{~cm}$ ). Similarly, the LL index was $1.12 \mathrm{~cm}$ on average (min: $0.88 \mathrm{~cm}$, max: $1.39 \mathrm{~cm}$ ). With these findings on the frontal and sagittal planes of the pelvis in the given specimens, it was possible to distinguish three pelvis shapes as "narrowing downwards", "cylindrical" and "expanding downwards" (Figure 1). The TT index was more than 1 and averaged from 1.06 to 1.5 in the narrowing downwards group (33.4\%), equal to 1 and ranged from 1 to 1.05 in the cylindrical group (50\%), and less than 1 (0.95-0.99) in the expanding downwards group (16.6\%). The LL index ranged between 1.06 to 1.39 in the narrowing downwards (12\%), 1 to 1.05 in the cylindrical $(63 \%)$ and 0.88 to 0.99 in the expanding downwards $(25 \%)$ groups.

The parameters were analyzed by distributing into groups according to the TT index to determine the shape of the pelvic cavity using the nonparametric Kruskal-Wallis test. This test allowed us to determine the dependent variables among the parameters (Table 2).
Accordingly, the shape of the pelvic cavity was dependent on the linear and angular parameters such as straight and transverse dimensions of the pelvic inlet and outlet, longitudinal size of the pelvic cavity, symphysis-tuberosity distance, subpubic angle, length of sacrospinous ligament, lumbosacral angle, and lower and medial angles of the obturator foramen.

To identify the morphometric parameters that have the maximum effect on the shape of the obturator foramen and to construct the predictive function, a discriminant analysis was carried out. A qualitative variable, the index of the obturator foramen, was used for grouping variables. The following variables were used as discriminant variables capable of influencing the shape of the obturator foramen: lower angle (Laof) of the obturator foramen, medial angle (Maof) of the obturator foramen, and the index of the obturator foramen. When planning the study, one of the objectives was to conduct a discriminant analysis for three assumed forms of the obturator foramen: triangular, ellipsoid and quadrangular. However, 
Table 2

Comparative characteristics of the morphometric parameters of the true pelvis and the obturator foramen in various forms of its cavity.

\begin{tabular}{|c|c|c|c|c|}
\hline \multirow[b]{2}{*}{ Pelviometric parameters $(\mathrm{cm}) /\left(^{\circ}\right)$} & \multicolumn{3}{|c|}{$\begin{array}{l}\text { Parameter values for various forms of } \\
\text { lesser pelvis (Mean } \pm \text { SD) }\end{array}$} & \multirow[b]{2}{*}{ p-value } \\
\hline & $\begin{array}{c}\text { Narrowing } \\
\text { downwards }(n=20)\end{array}$ & $\begin{array}{l}\text { Cylindrical } \\
(n=30)\end{array}$ & $\begin{array}{l}\text { Expanding } \\
\text { downwards }(n=11)\end{array}$ & \\
\hline Direct size of pelvic inlet & $9.1 \pm 0.8^{*}$ & $10.3 \pm 0.5$ & $11.5 \pm 0.4^{*}$ & $\begin{array}{c}p=0.002 \\
p_{1,3}=0.018\end{array}$ \\
\hline Direct size of pelvic outlet & $7.8 \pm 0.4^{*}$ & $8.6 \pm 0.2$ & $11.6 \pm 0.5^{\star}$ & $\begin{array}{c}p=0.004 \\
p_{1,3}=0.021\end{array}$ \\
\hline Longitudinal size of the pelvic cavity & $8.6 \pm 0.3^{*}$ & $9.2 \pm 0.5$ & $11.9 \pm 0.4^{*}$ & $\begin{array}{c}p=0.001 \\
p_{1,3}=0.011\end{array}$ \\
\hline Transverse size of the pelvic inlet & $11 \pm 0.5^{*},+$ & $12.7 \pm 0.3^{\dagger, \neq}$ & $13.1 \pm 0.3^{*}, \neq$ & $\begin{array}{c}\mathrm{p}=0.018 \\
\mathrm{p}_{1,2}=0.12 \\
\mathrm{p}_{1,3}=0.016 \\
\mathrm{p}_{2,3}=0.01\end{array}$ \\
\hline Transverse size of the pelvic outlet & $7.9 \pm 0.4^{*}, \dagger$ & $10.2 \pm 0.7^{\dagger}$ & $10.4 \pm 0.8^{*}$ & $\begin{array}{l}\mathrm{p}=0.0024 \\
\mathrm{p}_{1,2}=0.011 \\
\mathrm{p}_{1,3}=0.016\end{array}$ \\
\hline Symphysis-tuberosity distance & $8.2 \pm 0.3^{*}$ & $9 \pm 0.4$ & $10.3 \pm 0.3^{*}$ & $\begin{array}{l}p=0.003 \\
p_{1,3}=0.01\end{array}$ \\
\hline Pelvic height & $21.5 \pm 0.6$ & $19.4 \pm 0.4$ & $17.1 \pm 0.2$ & $p=0.081$ \\
\hline Height of pubic symphysis & $3.1 \pm 0.3$ & $3.9 \pm 0.1$ & $4.1 \pm 0.5$ & $p=0.75$ \\
\hline The pubic symphysis thickness & $1.2 \pm 0.3$ & $1.9 \pm 0.2$ & $2.1 \pm 0.2$ & $p=0.57$ \\
\hline Interspinal distance & $20.8 \pm 0.5$ & $22.6 \pm 0.4$ & $24.6 \pm 0.3$ & $p=0.21$ \\
\hline Width of pubic symphysis & $0.7 \pm 0.1$ & $0.9 \pm 0.2$ & $1.1 \pm 0.1$ & $p=0.15$ \\
\hline Interobturator foramina distance & $4.1 \pm 0.2$ & $5.1 \pm 0.4$ & $5.7 \pm 0.5$ & $p=0.20$ \\
\hline Subpubic angle ${ }^{\circ}$ & $93 \pm 2.2^{*,+}$ & $98 \pm 6.3^{t, \neq}$ & $106 \pm 3.1^{*, \neq}$ & $\begin{array}{c}\mathrm{p}=0.001 \\
\mathrm{p}_{1,2}=0.022 \\
\mathrm{p}_{1,3}=0.009 \\
\mathrm{p}_{2,3}=0.027\end{array}$ \\
\hline Length of sacrospinous ligament & $4.9 \pm 0.4^{*, \dagger}$ & $5.3 \pm 0.4^{\dagger, \ddagger}$ & $5.8 \pm 0.3^{*, \neq}$ & $\begin{array}{c}p=0.02 \\
p_{1,2}=0.02 \\
p_{1,3}=0.003 \\
p_{2,3}=0.001\end{array}$ \\
\hline Length of sacrotuberous ligament & $7.8 \pm 0.8$ & $8.2 \pm 0.4$ & $8.4 \pm 0.3$ & $\mathrm{p}=0.35$ \\
\hline Lower angle of the obturator foramen ${ }^{\circ}$ & $59.3 \pm 6.1 *,+$ & $66.8 \pm 8.2^{\dagger, \neq}$ & $75.3 \pm 5.1 *, \neq$ & $\begin{array}{c}\mathrm{p}=0.002 \\
\mathrm{p}_{1,2}=0.04 \\
\mathrm{p}_{1,3}=0.05 \\
\mathrm{p}_{2,3}=0.012\end{array}$ \\
\hline The medial angle of the obturator foramen ${ }^{\circ}$ & $77.4 \pm 3.4^{*},+$ & $85 \pm 4.1^{\dagger, \ddagger}$ & $90.1 \pm 4.9 *, \neq$ & $\begin{array}{c}\mathrm{p}=0.0013 \\
\mathrm{p}_{1,2}=0.031 \\
\mathrm{p}_{1,3}=0.011 \\
\mathrm{p}_{2,3}=0.04\end{array}$ \\
\hline Obturator foramen index & $0.55 \pm 0.3 *+\uparrow$ & $0.61 \pm 0.5^{\dagger, \ddagger}$ & $0.79 \pm 0.2^{*}, \neq$ & $\begin{array}{c}\mathrm{p}=0.043 \\
\mathrm{p}_{1,2}=0.028 \\
\mathrm{p}_{1,3}=0.016 \\
\mathrm{p}_{2,3}=0.03\end{array}$ \\
\hline The depth of the obturator foramen & $2 \pm 0.1$ & $2.2 \pm 0.1$ & $2.3 \pm 0.1$ & $p=0.32$ \\
\hline 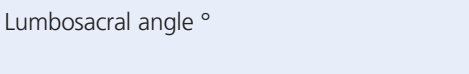 & $131 \pm 4.3^{*}$ & $136 \pm 2.1$ & $141 \pm 2^{*}$ & $\begin{array}{l}p=0.0051 \\
p_{1,3}=0.003\end{array}$ \\
\hline
\end{tabular}

* Relationship between narrowing downwards and expanding downwards; ${ }^{\dagger}$ relationship between narrowing downwards to cylindrical; ${ }^{\ddagger}$ relationship between cylindrical to expanding downwards.

the quadrangular form was found to be $3.3 \%$ (less than $5 \%$ of the total number of specimens), which was the criterion for its exclusion from the discriminant analysis.
Thus, the samples the distributed into two main groups as triangular (1) and ellipsoidal (0) forms of the obturator foramen (Table 3). 
The greatest influence on the shape of the obturator foramen was its lower angle. This angle is formed by the inferior pubic ramus and the ischial ramus. With the help of the data obtained, a discriminant function to calculate the shape of the obturator foramen was determined:

$$
\text { D0 }=0.416^{*} \text { Laof }+0.267^{*} \text { Maof }+22.97^{*} \text { OFI- } 66.562
$$

LA - low angle of the obturator foramen; MA - medial angle of the obturator foramen; OFI - index of the obturator foramen. If $\mathrm{D} 0>0$, the shape is considered as " 1 " (triangular), otherwise "0" (ellipsoid). With this formulation it was possible to assess the shape of the obturator foramen with above parameters with $96 \%$ sensitivity, $100 \%$ specificity and $97.1 \%$ accuracy.

While the correlational analysis of obturator foramen shape with the LL and TT index revealed a significant correlation with TT $(\mathrm{p}=0.053)$, there was no significant correlation with LL index ( $\mathrm{p}=0.12)$.

To determine the dependence of the shape of obturator foramen on the shape of pelvis, a statistical analysis was carried out according to the Pearson's criterion by creating a contingency table (Table 4). The results indicated that the triangular form was predominantly found in cylindrical and narrowing downwards group. In turn, the ellipsoid form dominated in the expanding downwards group. Due to the small sample number with a quadrangular shape of the obturator foramen, it was not possible to draw a certain conclusion about its relationship with the pelvic shape.

We also carried out a statistical analysis of standard parameters obtained in the course of a complex morphometric study in order to determine the correlation between linear and angular parameters of these pelvic bone forms according to the TT index. As a result, moderate direct correlational relations $(\mathrm{p} \leq 0.05)$ of the form narrowing downwards with the subpubic angle $(\mathrm{r}=74.9)$ were revealed, as well as its strong negative correlation $(\mathrm{p} \leq 0.05)$ with the lumbosacral angle $(\mathrm{r}=-0.86)$.

\section{Discussion}

Three forms of the obturator foramen were revealed: triangular, ellipsoid and quadrangular. The triangular shape of the obturator foramen was typically found in cylindrical or narrowing downwards configurations of the pelvis. At the same time, the ellipsoidal shape of the obturator foramen dominated in the expanding downwards configuration. On the basis of the direct correlation between the shape of the pelvis and the subpubic angle, it can be concluded that the pelvic cavity narrows
Table 3

Discriminatory variables and corresponding coefficients arranged in order of importance.

\begin{tabular}{lcc} 
Discriminatory variables & $\begin{array}{c}\text { Discriminatory } \\
\text { coefficient }\end{array}$ & p-value \\
\hline Lower angle of the obturator foramen & 0.416 & 0.002 \\
\hline Medial angle of the obturator foramen & 0.267 & 0.001 \\
\hline Obturator foramen index & 22.972 & 0.003 \\
\hline Constant & -66.562 & \\
\hline
\end{tabular}

downwards more when the subpubic angle gets smaller and vice versa.

A comprehensive analysis of the morphometric characteristics of the bone pelvis in adult women indicates a wide range of variant anatomy of linear and angular parameters and the size of the obturator foramen, which correlates with the results of former studies. ${ }^{[6,7]}$ Taking into account that the bone component of the obturator complex plays the role of a framework for fascial and muscular structures of the pelvis, its variability in shape can affect the position of implanted prostheses in tissues. ${ }^{[7]}$ Also, a significant role in understanding the distribution of the intra-abdominal pressure in the pelvis was played by the interconnection of the pelvic shape with the value of the lumbosacral angle, which increased significantly with the expansion of the pelvic cavity. Under such conditions, the pressure vector shifts from the spine to the pelvic diaphragm region, which can lead to anatomical and functional disorders of the pelvic floor. Our assumptions regarding the significance of the lumbosacral angle in the development of pelvic floor dysfunction are confirmed in the study of Stav et al..$^{[1]]}$ and Amonoo-Kuofi. ${ }^{[12]}$

The strong point of this study was the large number of samples used for determining pelviometric parameters and the use of discriminant analysis which turned out to be a sensitive statistical method and allowed to create a mathe-

Table 4

Occurrence of the obturator foramen forms depending on the shape of the pelvic cavity.

\begin{tabular}{lcccc} 
& \multicolumn{3}{c}{$\begin{array}{c}\text { Pelvic shape according to } \\
\text { transverse-transverse index }\end{array}$} \\
\cline { 2 - 5 } $\begin{array}{l}\text { Shape of the } \\
\text { obturator } \\
\text { foramen }\end{array}$ & Cylindrical & $\begin{array}{c}\text { Narrowing } \\
\text { downwards }\end{array}$ & $\begin{array}{c}\text { Expanding } \\
\text { downwards }\end{array}$ & Total \\
\hline Triangular & $23(59 \%)$ & $16(41 \%)$ & 0 & 39 \\
\hline Ellipsoid & $5(25 \%)$ & $4(20 \%)$ & $11(55 \%)$ & 20 \\
\hline Quadrangular & $2(100 \%)$ & 0 & 0 & 2 \\
\hline Total & 30 & 20 & 11 & 61 \\
\hline
\end{tabular}


matical model to predict the shape of the obturator foramen. We evaluated only the bone component of the obturator complex which was a limitation of our study. In order to confirm the given interrelations, it is necessary to find out the role of the obturator muscles and the obturator membrane, as well as to study the morphology of the paraurethral area. This collection did not have medical history data or information on parity or pelvic floor disorders, therefore, we could not comment on the relationship between these findings and clinical outcomes. Furthermore, some of these bony pelvises had mild degeneration that could potentially affect the measurements.

\section{Conclusion}

There is a need to study individual pelvic anatomy in the surgical treatment of SUI. The obturator foramen and angle of the pubic arch are highly variable in females. It is important to take into account that the bone component of the obturator complex is a framework for fascial and muscular structures of the pelvis, and the role of individual anatomy of muscles and ligaments in the studied area for the successful surgical treatment of stress urinary incontinence in women and should be evaluated in further studies. The results of this study did not answer the questions for improving the objective efficacy of suburethral sling, but suggested further studies using imaging techniques and cadaver dissections.

\section{Acknowledgements}

The authors wish to sincerely thank Ekaterina Shapovalova, Natalia Nichiporuk, Anastasiya Zaitseva and the staff of the «Military Medical Academy named after S.M. Kirov» of the Ministry of Defense of the Russian Federation for supporting this research and those who donated their bodies to science so that anatomical research could be performed. Results from such research can potentially improve patient care and increase mankind's overall knowledge. Therefore, these donors and their families deserve our highest gratitude.

\section{Author Contributions}

Research concept and design: GIV, SDD; collection and processing of material: KGV, LIA, NGI; data analysis and interpretation: $\mathrm{KGV}, \mathrm{KND}$; writing and editing text: KGV, LIA.

\section{References}

1. DeSilva JM, Rosenberg KR. Anatomy, development and function of the human pelvis. Anat Rec (Hoboken) 2017;300:628-32.

2. Betti L. Human variation in pelvic shape and the effects of climate and past population history. Anat Rec (Hoboken) 2017;300:687-97.

3. Musielak B, Kubicka AM, Rychlik M, Czubak J, Czwojdzinski A, Grzegorzewski A, Jóêwiak M. Variation in pelvic shape and size in Eastern European males: a computed tomography comparative study. PeerJ 2019;7:e6433.

4. Maggiore U, Agrò E, Soligo M, Li Marzi V, Digesu A, Serati M. Long-term outcomes of TOT and TVT procedures for the treatment of female stress urinary incontinence: a systematic review and meta-analysis. Int Urogynecol J 2017;28:1119-30.

5. Ford AA, Rogerson L, Cody JD, Aluko P, Ogah JA. Mid-urethral sling operations for stress urinary incontinence in women. Cochrane Database Syst Rev 2017;7:CD006375.

6. Ridgeway BM, Arias BE, Barber MD. Variation of the obturator foramen and pubic arch of the female bony pelvis. Am J Obstet Gynecol 2008;198:546.e1-4.

7. Bogusiewicz M, Rosinska-Bogusiewicz K, Drop A, Rechberger T. Anatomical Anatomocal variation of bony pelvis form the viewpoint of transobturator sling placement for SUI. Int Urogynecol J 2011; 22:1005-9.

8. Handa VL, Pannu HK, Siddique S, Gutman R, VanRooyen J, Cundiff G. Architectural differences in the bony pelvis of women with and without pelvic floor disorders. Obstet Gynecol 2003;102: 1283-90.

9. Greulich WW, Thoms H. A study of pelvic type. JAMA 1939;112: 485.

10. Handa VL, Lockhart ME, Fielding JR, Bradley CS, Brubaker L, Cundiff GW, Ye W, Richter HE; Pelvic Floor Disorders Network. Racial differences in pelvic anatomy by magnetic resonance imaging. Obstet Gynecol 2008;111:914- 20.

11. Stav K, Alcalay M, Peleg S, Lindner A, Gayer, G, Hershkovitz I. Pelvis architecture and urinary incontinence in women. Eur Urol 2007;52:239-44.

12. Amonoo-Kuofi HS. Changes in the lumbosacral angle, sacral inclination and the curvature of the lumbar spine during aging. Acta Anat (Basel) 1992;145:373-7.
ORCID ID:

I. V. Gaivoronskiy 0000-0003-2531-3807; I. A. Labetov 0000-0001-9813-7483; G. V. Kovalev 0000-0003-4884-6884; G. I. Niciporuk 0000-0001-5569-7325; N. D. Kubin 0000-0001-5189-4639; D. D. Shkarupa 0000-0003-0489-3451

\author{
Correspondence to: Ivan Antonovich Labetov, MD \\ Fontanka River Embankment, 154., \\ 190005, Saint Petersburg, Russia \\ Phone: +7 9313070139 \\ e-mail: ivanlabetov@gmail.com
}

Conflict of interest statement: No conflicts declared.

This is an open access article distributed under the terms of the Creative Commons Attribution-NonCommercial-NoDerivs 3.0 Unported (CC BY-NC-ND3.0) Licence (http://creativecommons.org/licenses/by-nc-nd/3.0/) which permits unrestricted noncommercial use, distribution, and reproduction in any medium, provided the original work is properly cited. Please cite this article as: Gaivoronskiy IV, Labetov IA, Kovalev GV, Niciporuk GI, Kubin ND, Shkarupa DD. Relationship between the shape of the obturator foramen and the shape of the pelvic cavity in adult women. Anatomy 2020;14(1):53-60. 